\title{
Superconducting Magnets
}

\section{4}

Accelerator and Fusion Research Division Lawrence Berkeley Laboratory

University of California

1 Cyclotron Road

Berkeley, CA 94720

August 1994

Reprinted from Accelerator and Fusion Research Division 1993 Summary of Activities, LBL-35476 (April 1994)

This work was supported principally by the U.S. Department of Energy under Contract No. DE-AC03-76SF00098. Support came from the Director, Office of Energy Research, through the Office of High Energy and Nuclear Physics (High Energy Physics Division) and the Office of Superconducting Super Collider. 



\section{DISCLAIMER}

This report was prepared as an account of work sponsored by an agency of the United States Government. Neither the United States Government nor any agency thereof, nor any of their employees, make any warranty, express or implied, or assumes any legal liability or responsibility for the accuracy, completeness, or usefulness of any information, apparatus, product, or process disclosed, or represents that its use would not infringe privately owned rights. Reference herein to any specific commercial product, process, or service by trade name, trademark, manufacturer, or otherwise does not necessarily constitute or imply its endorsement, recommendation, or favoring by the United States Government or any agency thereof. The views and opinions of authors expressed herein do not necessarily state or reflect those of the United States Government or any agency thereof. 


\section{DISCLAIMER}

Portions of this document may be illegible in electronic image products. Images are produced from the best available original document. 


\section{Table of Contents}

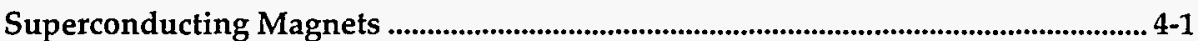

Advanced Lattice Magnets: Higher Fields, Lower Cost ............................................4-4

D19B and -C: The Next Steps for a Record-Setting Magnet .............................. 4-4

D20: The Push Beyond $10 \mathrm{~T}$.................................................................................... $4-6$

Beyond D20: Speculations on the 16-T Regime ...............................................4-7

Other Advanced Magnets for Accelerators …......................................................4-7

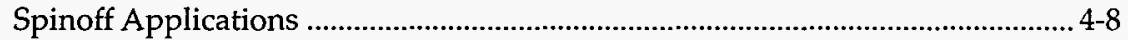

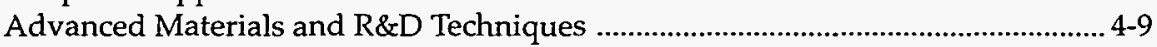

APC Materials Development ............................................................................. 4-9

Cable and Cabling-Machine Development ...................................................... 4-12

High- $T_{c}$ Superconductor at Low Temperature ................................................. 4-13

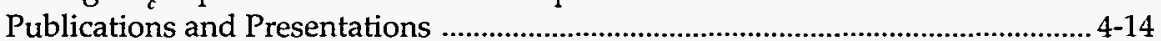


S

UPERCONDUCTING MAGNETS PLAY VITAL ROLES AT THE frontiers of particle accelerator performance. They also have many nonaccelerator applications, ranging from mangetic-resonance imaging to magnetically levitated trains. Focusing primarily on accelerators, the Superconducting Magnet Program in AFRD takes a vertically integrated approach by offering expertise "from melt to magnet" - that is, at all phases from basic development of better superconducting materials to evaluation of finished magnets.

Interaction with private industry is a vital part of our activities. Even when the goal is a magnet design for a particle accelerator, industrial partnership is important because the sheer size of today's large colliders mandates industrial production. But in many areas, especially in basic materials research and development, our work goes beyond "technology transfer" and into the realm of genuine two-way collaboration.

In 1993 our program continued its long-term shift toward generic R\&D that will benefit future accelerators for high-energy and nuclear physics. Earlier we had set a field-strength record for accelerator-type magnets: just over $10 \mathrm{~T}$ in the magnet $\mathrm{D} 19$, made with niobium-titanium superconductor. That performance level, however, appears to be near the practical limit for niobium-titanium. While working to enhance the performance of $\mathrm{NbTi}$ through the use of artificial flux-pinning centers, we are exploring other materials that are not currently as well-proven and economical as niobium-titanium but hold the promise of higher fields (and possibly lower costs as well) with further R\&D. They include brittle materials such as niobium-tin with and without artificial pinning centers.

Innovative approaches are also being taken in ancillary fields. For example, a new type of strain gage, using fiber optics to measure strain through optical interferometry, has passed fundamental proof-of-principle tests, and we are now working on ways to incorporate it in magnets and to implement it as part of an integrated circuit chip. And, after three years, our research into "high-temperature" bismuth oxide superconductor is approaching its first macroscopic demonstration: a small test solenoid. Although far from being ready for use as an engineering material in high-field accelerator magnets, this high- $\mathrm{T}_{\mathrm{c}}$ material holds great promise for further exploration and industrial spinoffs. 


\section{Capabilities and Facilities}

The Superconducting Magnet Program has expertise in all aspects of design and evaluation "from melt to magnet." In many years of collaboration with users, academic researchers, and industrial partners, we have amassed a track record of success that includes:

- Improved superconducting materials.

- Manufacturing of cable from superconducting wire and design of improved machines for cabling.

- Design of superconducting and normal-conducting magnets to meet a variety of needs.

- Computerized analysis of magnetic and mechanical properties expected from complex magnet designs.

- Testing and magnetic measurement, along with related instrumentation.

- Transfer of designs and fabrication skills to industry*

The program is also supported extensively by other LBL organizations, especially the Engineering Division.

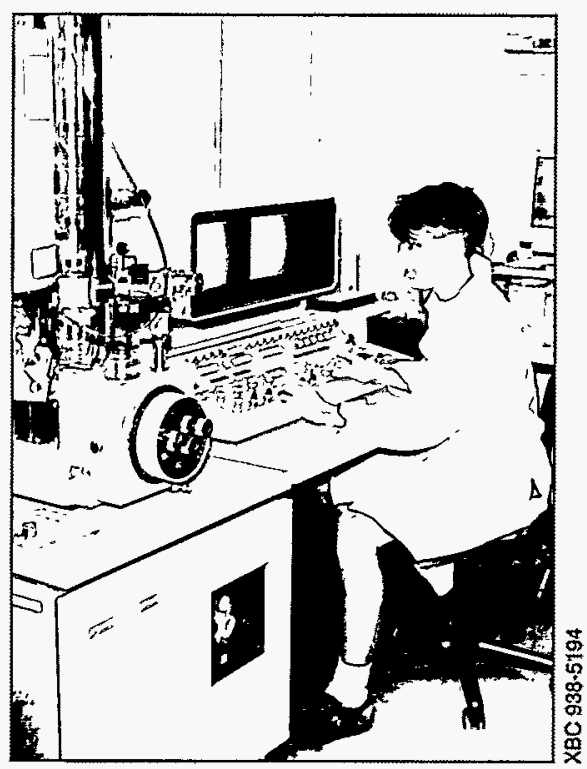

A scanning electron microscope with 3$\mathrm{nm}$ resolution and analytical capabilities is the latest addition to a dedicated materials-testing laboratory*

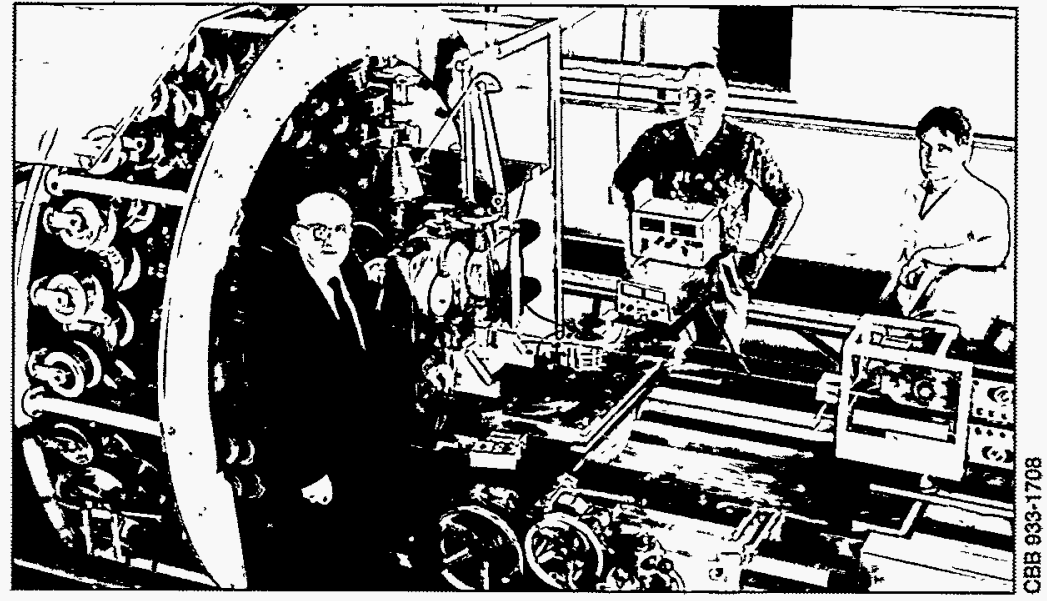

This machine has fabricated superconducting cable of up to 60 strands with keystoned, rectangulax, or other profiles.
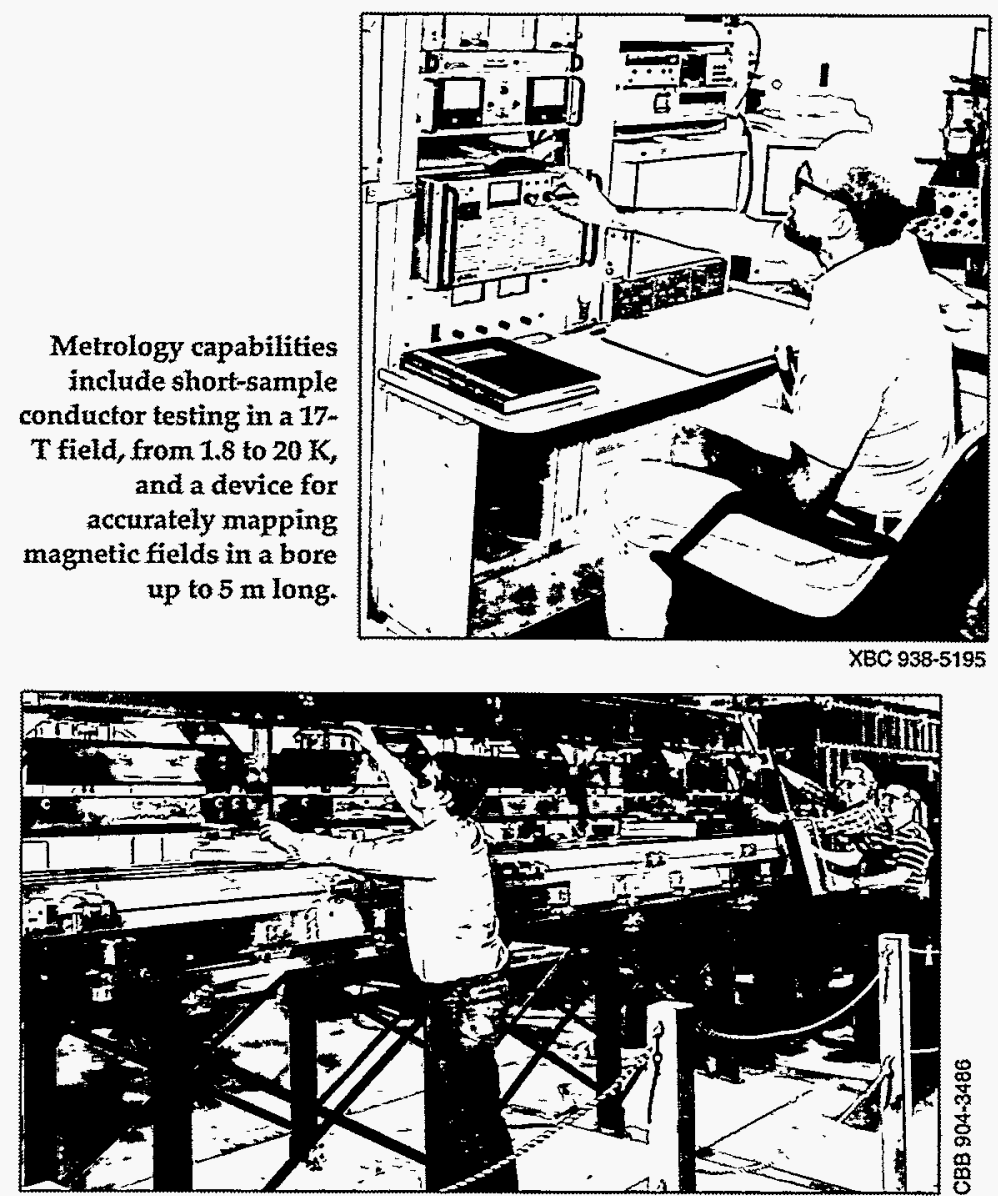

Development of manufacturable magnets and transfer of technology to industry are among the proven strengths of the program. 
Applied development work for the Superconducting Super Collider dominated our activities for several years, but we continued to investigate other aspects of superconducting-magnet science and technology. The findings will be increasingly relevant to new and upgraded accelerators (especially colliders for high energy and nuclear physics) as their designers attempt to minimize costs, build advanced accelerators in existing tunnels, and face challenges such as final focus of energetic beams in the constrained spaces in and around detectors.

Our quest for higher fields in accelerator-style magnets has taken us in two directions. Niobium-titanium (NbTi) material has been used in most practical superconducting magnets to date because it is ductile and therefore workable in its superconducting form. In close collaboration with an industrial partner, Supercon, Inc., we are industrializing Artificial Flux Pinning Center (APC) technology. This approach to making $\mathrm{NbTi}$ superconductor with artificial flux pinning centers promises considerable improvements in critical current density at high magnetic fields, a key measure of performance, and, at the same time, cost reductions of $40 \%$ or more. (For higher fields, we are also experimenting with a ductile ternary alloy, niobium-titanium-tantalum or NbTiTa.) This combination of higher performance and lower cost will give NbTi superconductor many years of continuing usefulness.

In the long run, though, NbTi will reach a practical limit in the neighborhood of $10 \mathrm{~T}$. To push accelerator magnets to much higher fields, we are revisiting various brittle materials, such as niobium-tin $\left(\mathrm{Nb}_{3} \mathrm{Sn}\right)$, that were investigated in the 1970s but neglected in favor of the easier-to-fabricate NbTi. These materials present great challenges in cable-making and in magnet fabrication that are quite different from those of ductile superconductors. Our present thrust is toward $13 \mathrm{~T}$ with $\mathrm{Nb}_{3} \mathrm{Sn}$. On the drawing board is a magnet intended to reach $16 \mathrm{~T}$-possibly by using the APC approach in brittle material such as $\mathrm{Nb}_{3} \mathrm{Sn}$ or other A15 superconductors, assuming that we and our academic and industrial collaborators can make such materials.

To advance magnet performance and to support our development of superconducting cables and the machines to make them, we build experimental magnets comparable in size and shape to those used in accelerators. The aforementioned field-strength record for such magnets of $10.06 \mathrm{~T}$ central field (approximately $10.4 \mathrm{~T}$ near the conductor) ${ }^{*}$ was set using D19, a high-field dipole.

D19 uses the same NbTi cables as the SSC collider dipole magnets: 30 strands for the inner layer of windings, 36 in the outer. The field record was set at $1.8 \mathrm{~K}$, with a current of about $9.4 \mathrm{kA}$. With its more efficient coil and yoke design, D19 reached the SSC-specified dipole field of $6.6 \mathrm{~T}$ at $5.8 \mathrm{kA}$ and $4.3 \mathrm{~K}$, a considerable improvement over the $6.6 \mathrm{kA}$ of the SSC dipole. The cross section in Figure 4-1 reveals the noncircular inner profile of the iron yoke. The use of this profile, an existing idea not previously applied to accelerator-type magnets, maximizes the magnetic-field contribution of the iron while keeping high-field saturation effects down to reasonable levels. Since then, we have been evaluating improved superconducting materials for accelerator service by building and testing new variations on the D19 design.

\section{Advanced Lattice Magnets: Higher Fields, Lower Cost}

\author{
D19B and -C: The Next \\ Steps for a Record- \\ Setting Magnet
}

\footnotetext{
"The record was recently edged by a group at CERN, the European high-energy physics laboratory.
} 
Figure 4-1. Dipole D19, the 10-T magnet now being used for APC testing, has an iron yoke with a noncircular profile for efficiency. This shape maximizes the magnetic-field contribution of the iron while keeping high-field saturation effects down to manageable levels. Another interesting feature is the vertically split iron yoke with aluminum-alloy spacers between the halves. The spacers maintain a predetermined gap between the halves at room temperature but allow them to shrink together tightly at cryogenic temperatures. This maintains the high compressive load on the coils and prevents the windings from shrinking faster than the iron yoke during cooldown. Such differential contraction would relieve the compression of the windings and thus undo the "training" process that allows the ultimate magnetic field to be reached.
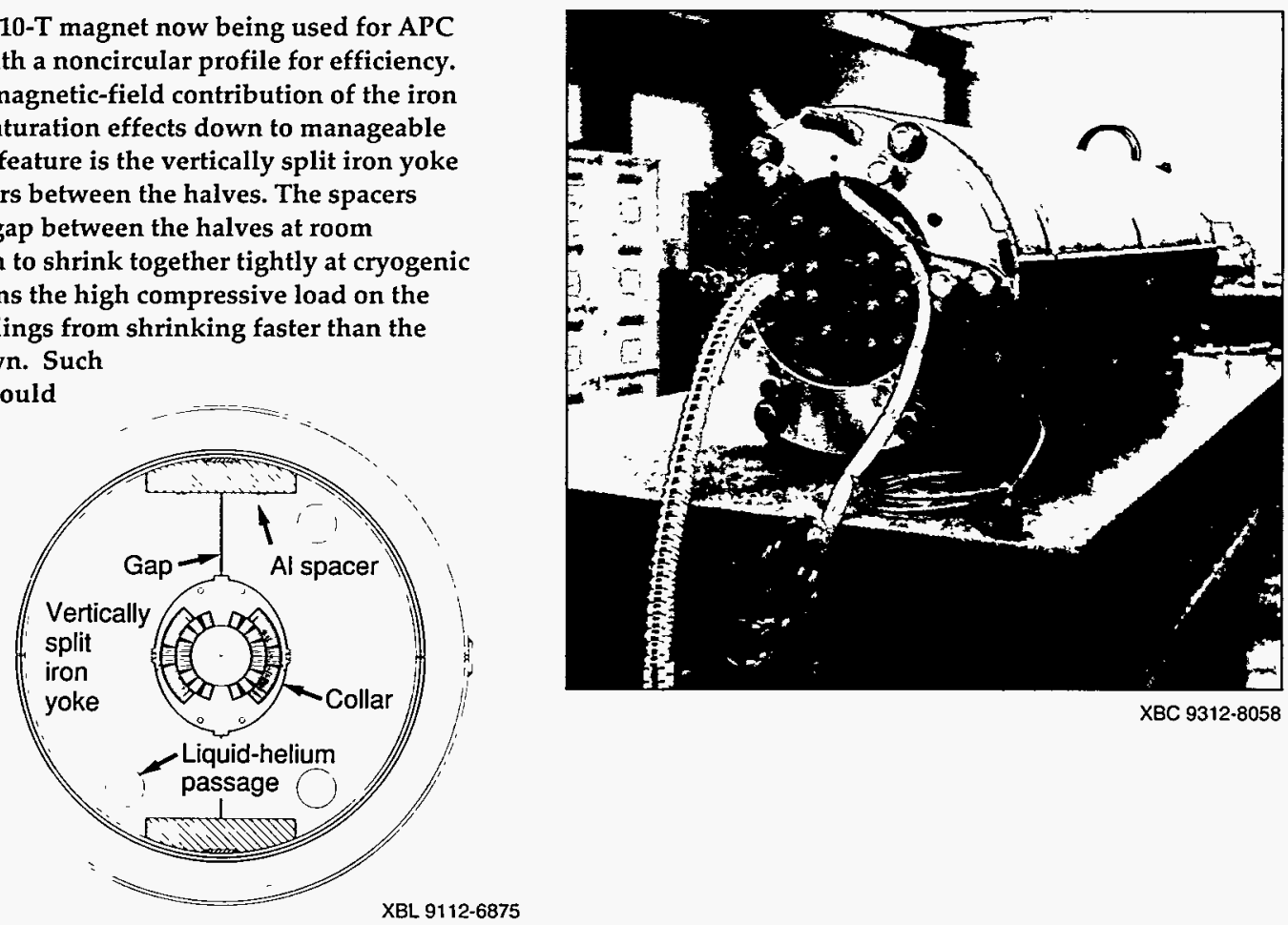

XBC $9312 \cdot 8058$

In 1993 we completed and began testing D19B, a follow-on project using the same design but incorporating superconductor that has artificial fluxpinning centers for higher critical current. This APC superconductor (whose principles and fabrication are explained in the later section on materials R\&D) was produced by one of our frequent industrial collaborators, Supercon, Inc., with a novel "jelly-roll" process. Figure 4-2 shows training curves for D19 and its successor.

Figure 4-2. This graph shows the training behavior of dipole $\mathrm{D} 19 \mathrm{~B}$, along with its record-setting predecessor D19. After six training quenches, D19B, made with APC conductor from Supercon, Inc., plateaued at a magnetic field of $7.0 \mathrm{~T}$ at a temperature of $4.3 \mathrm{~K}$ (lower set of curves). Subsequently it reached $8.7 \mathrm{~T}$ at $1.8 \mathrm{~K}$ (upper set of curves). The dotted line in each half of the drawing indicates the maximum possible magnetic field predicted from critical current density measurements of the conductor and short samples of the cable. The various shapes of points indicate which of the four coils experienced the quench that ended each test. The plateau field observed was $97 \%$ of the field predictedan excellent achievement for the first sample of a new type of cable.

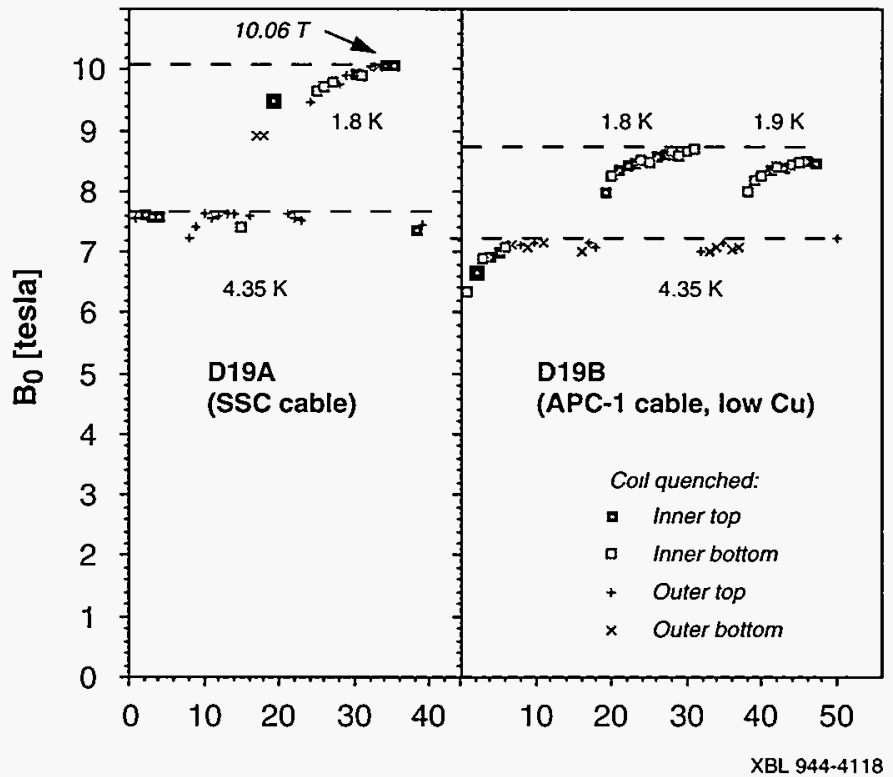


Although D19B's performance reached a plateau at a considerably lower field than D19, it was a success. The main goals were not to set a new field record, but rather to demonstrate that a new material from a new process was qualitatively similar in behavior to the familiar NbTi materials (measurements thus far indicate that this is indeed the case) and to gain knowledge that will be useful as the materials process moves further from laboratory work to full manufacturing scale. Materials produced in the full-scale process will be evaluated in D19C, the next step in this part of the program.

After that, D19D will give us our first look at one of the higher-field ternary (three-part) alloys, NbTiTa, with artificial pinning centers. This project has been delayed somewhat because of difficulties in getting the constituent materials in the form needed for the jelly-roll process, but work is expected to get under way in 1994.

Another advanced magnet, D20, is now being fabricated (Figure 4-3). D20 will press through the 10-T level, and hopefully well beyond, by using $\mathrm{Nb}_{3} \mathrm{Sn}$ superconductor. The goal is a "short-sample" field of about $13 \mathrm{~T}$ at

D20: The Push Beyond $10 \mathrm{~T}$ $1.8 \mathrm{~K}$. (The short-sample field represents the maximum performance of the superconducting cable and is determined by measuring the conductivity of a short sample in an external magnetic field. An actual magnet incorporating the cable might, if designed and constructed well, approach the shortsample limit near the edge of the aperture, where the field is strongest.)

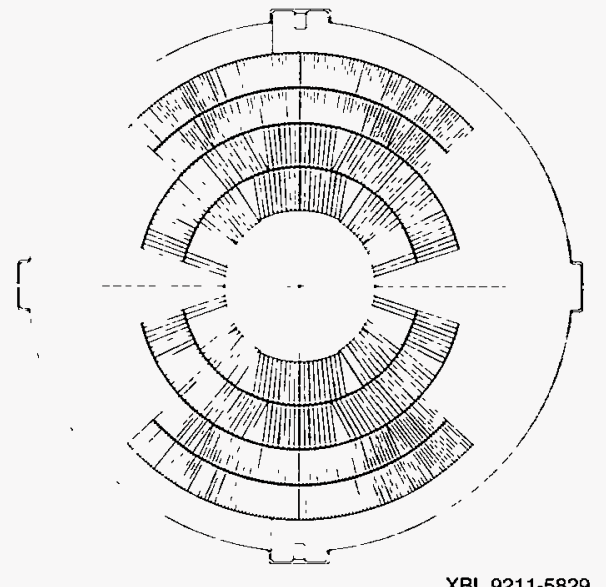

The effort to reach beyond $10 \mathrm{~T}$ in accelerator-type magnets presents a number of challenges above and beyond magnetic design. Mechanical stresses become far greater than those in existing accelerator magnets: at 13 $\mathrm{T}$, the windings in D20 would experience an axial force of about 820000 newtons $(185000 \mathrm{lb})$, a horizontal force of $3900 \mathrm{kN} / \mathrm{m}(23000 \mathrm{lb}-\mathrm{in}$.$) , and a$ compressive stress of $100 \mathrm{MPa}(15000 \mathrm{psi})$. Such large forces exerted on a precision assembly of modest size can have a variety of adverse effects. In its most severe form, the strain can distort the magnet and damage the brittle superconductor. Short of that level, it can cause the small movements of the cable that lead to quenches, and can reduce the critical current of the superconductor, an effect that we are continuing to investigate. 
Beyond D20: Speculations on the 16-T Regime
Other Advanced Magnets for Accelerators
Even more important, $\mathrm{Nb}_{3} \mathrm{Sn}$ in its finished, superconducting form is brittle, as are all the other likely candidates for future high-field magnets. Cable containing the ingredients-still ductile at this point-is wound onto magnet forms and then baked at $650^{\circ} \mathrm{C}$ for about two weeks. The heating causes a chemical reaction that turns it into superconductor and also embrittles it. This magnet-making procedure is essentially a new technology. It is important because, although ductile ternary compounds like $\mathrm{NbTiTa}$ might offer small improvements over $\mathrm{NbTi}$, the high-field frontier clearly belongs to brittle superconductors. They hold the potential for current densities much greater than that of $\mathrm{NbTi}$, which is considered to have a theoretical limit in the neighborhood of $5000 \mathrm{~A} / \mathrm{mm}^{2}$ at $5 \mathrm{~T}$ and a practical limit of rather less than that.

Cabling, another important part of our vertically integrated capabilities, also plays an important role in the D20 project. Previous accelerator magnets from recent years had generally used "Rutherford-style" cable with a keystoned cross section, which allows robust Roman-arch construction of the magnet. However, to build a magnet that can withstand the extreme Lorentz forces of high-field operation, considerable pressure has to be applied mechanically during fabrication. This causes compaction of the superconductor, which in turn results in some degradation of critical current density $\left(J_{\mathrm{c}}\right)$.

Typically in the past, with $\mathrm{NbTi}$, the degradation had only been about $5 \%$, but as we studied cabling of $\mathrm{Nb}_{3} \mathrm{Sn}-\mathrm{a}$ subject that, as it turned out, had never been examined closely - we realized that the $J_{\mathrm{C}}$ penalty in D20 could approach $40 \%$. Thus we went back to a rectangular profile for the D20 cable, changing the cross section of the magnet accordingly.

Although D20 is intended solely for testing $\mathrm{Nb}_{3} \mathrm{Sn}$, it has many features that are generically applicable to future hadron colliders. The high field permits an accelerator to achieve higher energies in a given radius, as when upgrading a collider or building a new one in an existing tunnel.

Thus far, the familiar cosine-theta coil configuration, held in compression when idle and fortified against stresses during operation solely by an external yoke, has sufficed for our advanced magnets. Eventually, as we push the magnetic field strength into the high teens and accordingly increase the stresses and resulting strains inside the windings, we may need to use alternative designs for coils and cables. The problems are more severe in brittle superconductors than in $\mathrm{NbTi}$ because $\mathrm{J}_{\mathrm{c}}$ is more rapidly degraded by strain: a strain of $0.7 \%$ is considered to be the limit, which must be obeyed everywhere in the magnet. Accordingly, we are considering some speculative designs, such as the "block" and "window-frame" configurations of Figure 5-4. At the appropriate time, such a design will be chosen, modeled in detail, and implemented in a future magnet. Building upon the D20 cable development work, we are also examining possibilities for cable made with other strand cross sections that may reduce strain degradations.

One of the challenges of the high-energy, high-luminosity colliders of the future, such as the PEP-II B-meson "factory" recently approved for construction at the Stanford Linear Accelerator Center, is final focus of the beams just before the interaction point. (Various other beam manipulations may also be needed in these areas.) These and other magnets in and around the detectors 


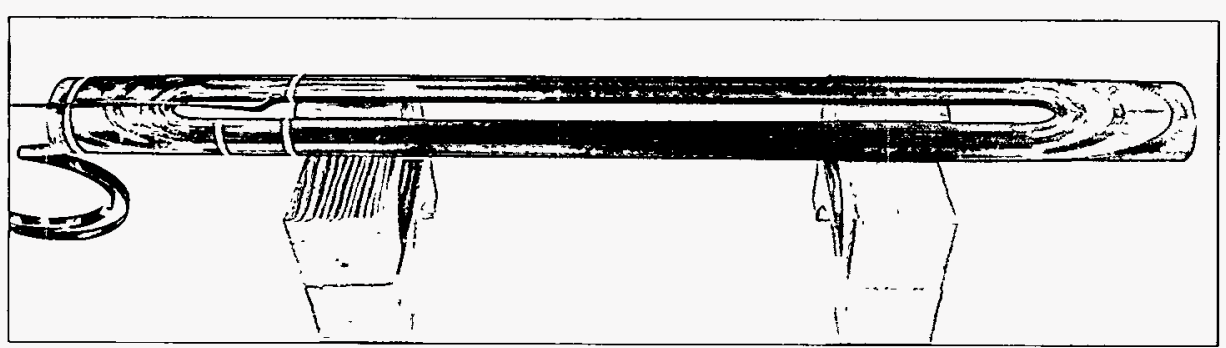

CBB 915-4055
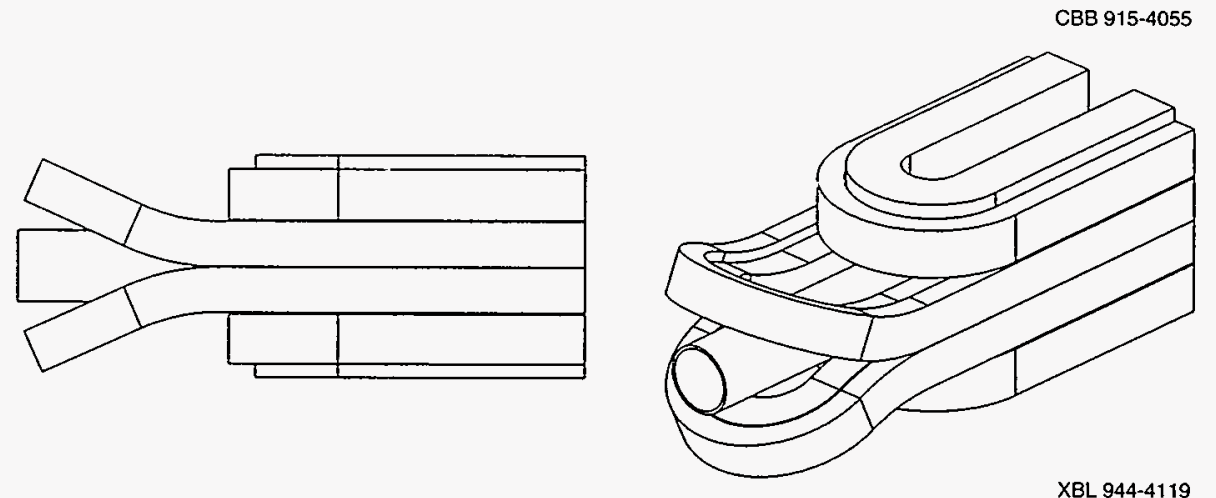

are usually quite challenging in shape and size as well as in field strength. We are working with the PEP-II collaboration to design and develop finalfocus magnets and other advanced superconducting and normal-conducting magnets that might be needed. See Chapter 3, "Center for Beam Physics," of the 1993 AFRD Summary of Activities for further information on PEP-II.

Although our program grew up alongside high-energy physics and is strongly oriented toward service to the accelerator community, there are many other applications for high-field superconducting magnets. One of them is the field of nuclear magnetic resonance (NMR) and magneticresonance imaging (MRI). ${ }^{*}$ In a collaborative project with Wang NMR of Livermore, California and the Bitter National Magnet Laboratory at the Massachusetts Institute of Technology, we developed the conductor for a gigahertz NMR spectrometer at Pacific Northwest Laboratory.

This proton spectrometer would be part of the Molecular Science Research Center, an initiative proposed by Pacific Northwest Laboratory, and would be especially well suited to studying the large molecules and systems of molecules that are of interest to biologists. The spectrometer design requires a large 23.5-T magnet. ${ }^{\dagger}$ Large magnets for NMR spectrometers are also of interest to the National High Magnetic Field Laboratory at Florida State University.

-Generally speaking, NMR refers to the nonimaging scientific tool and to the underlying physical principle, whereas MRI refers to the imaging technique used for applications such as medical diagnosis.

t Note that the 10-T record mentioned earlier was for an accelerator-type magnet. The magnets for high-energy accelerators must exert their field over a considerable length in order to bend or focus the extremely "rigid" particle beam, and the field must be extremely homogeneous. Their design is also constrained by many mechanical and economic factors. NMR magnets, although challenging in their own way, are much different physically, so the apparent tremendous leap from $10 \mathrm{~T}$ in accelerator magnets to $23.5 \mathrm{~T}$ in this project is misleading.
Figure 4-4. Most superconducting accelerator magnets thus far have used "cosine-theta" windings, as revealed in this photograph of D19 at an intermediate stage of assembly. In speculative designs for accelerator-style magnets that will reach beyond $13 \mathrm{~T}$ or so, we are considering a variety of alternate configurations, like the one shown, that may give the desired type and uniformity of magnetic field while minimizing the strain that results from the high stresses in these strong magnets. Building upon such concepts, the design process will involve detailed, computerized magnetic and mechanical modeling.

\author{
Spinoff Applications
}




\section{Advanced Materials and R\&D Techniques}

\author{
APC Materials \\ Development
}

In addition to designing and building magnets, we conduct a diverse program of investigations in superconducting materials. Current projects explore the use of artificial flux pinning center (APC) technology in ductile and brittle materials and investigate still-embryonic but promising low-temperature applications of high-T $T_{C}$ superconductors. Other projects include a novel interferometric method of measuring strain in magnets; it uses optical fiber not only in its conventional role as a "light pipe" for imaging or information, but also as the main element of a physical sensor.

Materials-science research plays a key role in achieving higher, more uniform, and more predictable magnetic fields-for example, a promising recent line of inquiry that involves the APC concept. In APC superconductors, the random distribution of pinning centers, which ordinarily arise from alpha-titanium precipitation when the superconductor is formed, is replaced with a moreprecise distribution. Ideally the distribution matches the magnetic fluxoid lattice for a given field strength: $25 \mathrm{~nm}$ for $5 \mathrm{~T}$, for example. The optimum diameter is roughly twice the electron coherence length, or about $11 \mathrm{~nm}$.

A fluxoid is the site of one quantum of magnetic effect and may be thought of as the place where a line of magnetic flux penetrates the superconducting wire. Ordinarily, fluxoids can move through the superconductor in response to an applied magnetic field; this dissipates energy. In one approach to APC wire, niobium is introduced as a normal-conducting phase during wire manufacturing. The flux lines are localized to the regions where this material remains after the "jelly roll" of wire has been drawn down to the final size. Artificial flux pinning allows some measure of control over the final microstructure of the superconducting material, which is important because microstructure is an intrinsic characteristic that cannot be altered by the way the material is formed into wires. (The significant intrinsic factors are filament microstructure and composition. Extrinsic factors, such as the cross-sectional area, integrity, and uniformity of the superconductor, are also important.) Figure 4-5 illustrates this principle.

The ultimate application of this research is to enable the fabrication of multifilamentary superconducting wire that has higher $J_{c}$ and is also more economical to produce. We have also worked with Supercon, Inc., on a method (Figure 4-6) of producing APC superconductor from sheets of $\mathrm{Nb}$ and Ti metal. This method improves the high-field performance of the APC superconductor and also promises to reduce the cost of the material.

After successful demonstrations, Supercon, Inc. scaled up to the wire sizes appropriate for SSC-type dipole magnets, and we obtained enough of the wire to fabricate cable and build the D19B magnet described in the previous section. Testing this 1-m-long dipole magnet gave us further information on the performance, reliability, and cost-effectiveness of APC material.

Superconducting magnets retain the desired electrical properties only up to a certain critical current; above that level, they regress to ordinary conduction. Current density, temperature, and magnetic field interact in this regard; the superconducting regime is often graphed along three axes and referred to as the " $J_{c}, T_{c}, B_{c}$ surface." $B_{c}$ and $J_{c}$ can be limited by fundamental properties of the superconductor; however, $J_{c}$ can be increased by optimizing the microstructure. Increases in $J_{c}$ allow the use of smaller, cheaper cable or, alternately, the achievement of higher fields. 


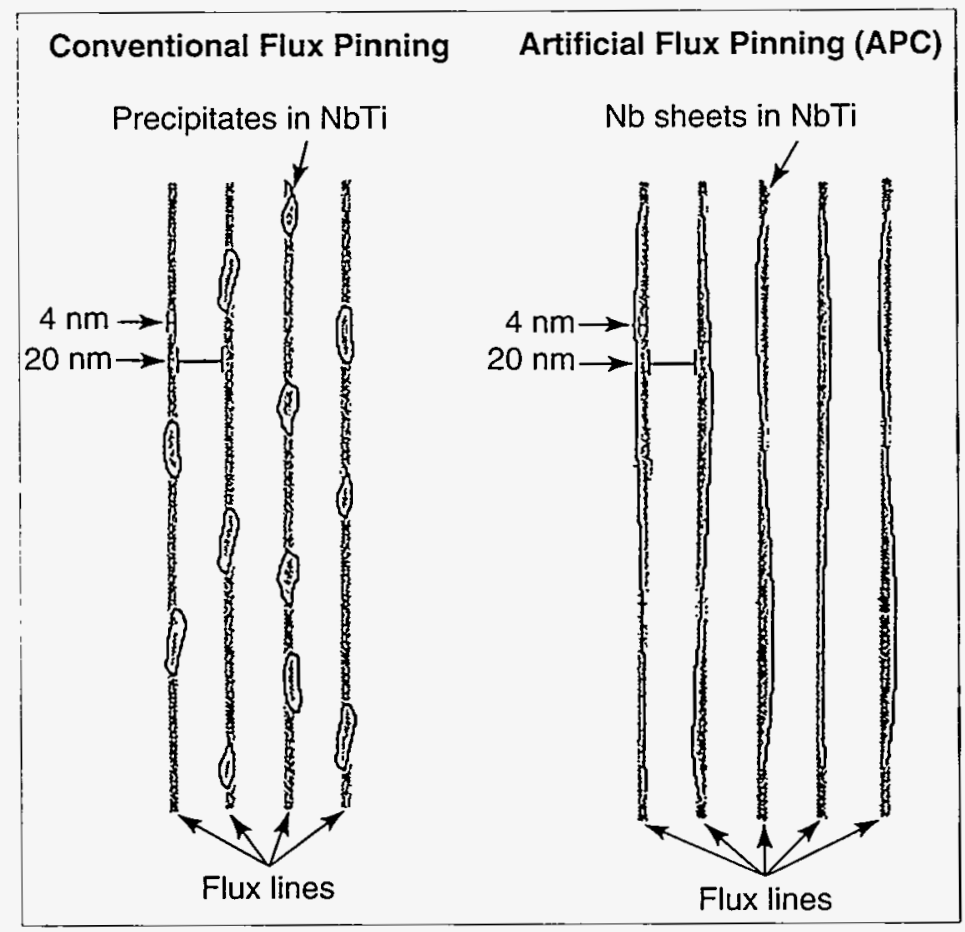

Figure 4-5. In conventional flux pinning (left), the fluxoids, or places where field lines penetrate the conductor, are localized at the randomly distributed points where precipitates were formed by heat treatment. (These sites are elongated, as shown here, when the material is drawn into wire.) In artificial flux pinning, normal-conducting metal sheets are interleaved with the superconductor in the manufacturing process and drawn down to nanometer scale. This process provides better matching between the pinning sites and the flux lines. It also obviates the formation of pinning centers as a reason for heat treatment.

XBL $9211-5830$
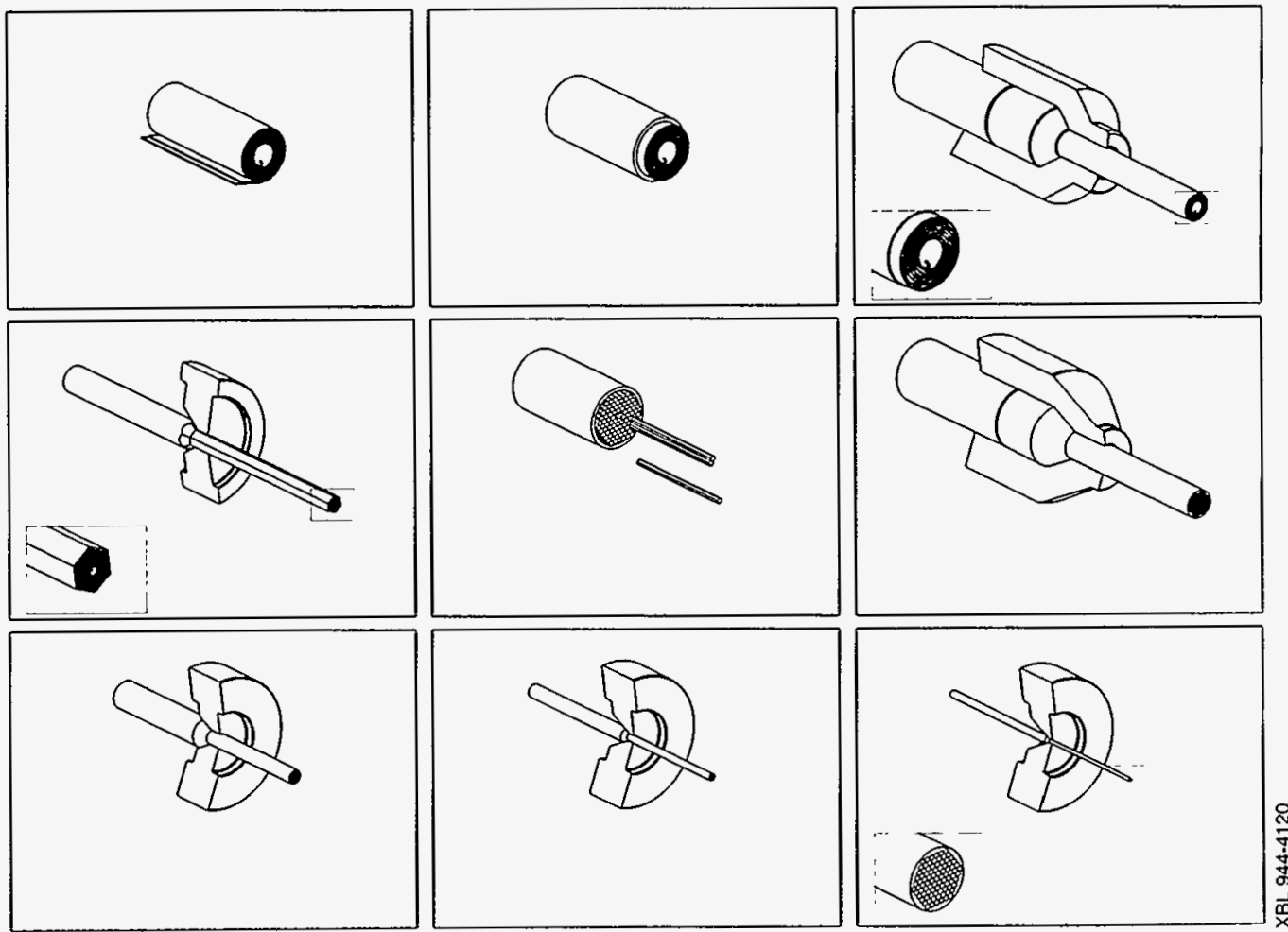

Figure 4-6. This conceptual diagram of the APC manufacturing process patented by Supercon, Inc., shows how a "jelly roll" of alternating $\mathrm{Nb}$ and $\mathrm{Ti}$ sheets (1) is loaded into a Cu can (2), extruded into a monofilament (3), and drawn through a hex die and cut into stackable lengths (4), which are then loaded into a can (5), which is extruded into a multifilamentary billet (6) and successively drawn into wire of the final diameter (7-9). Because no heat is applied during the process, the copper matrix remains unannealed (avoiding the introduction of large differences in mechanical properties between matrix and filament), and interdiffusion at the filament boundary is obviated. 
The D19B wire-essentially the first example of a recently scaled-up production process — exhibited some filament "sausaging": about $12.5 \%$, as opposed to the $6 \%$ typical of the final SSC production runs. (Sausaging is quantified by dividing the average diameter into the standard deviation after digital measurement of filament sizes in cross section.) The sausaging is attributed to a slightly higher ratio of inter-filament spacing to filament diameter $(0.21$ rather than the specified 0.17$)$. This caused the average $J_{c}$ $\left(2200 \mathrm{~A} / \mathrm{mm}^{2}\right)$ to be slightly lower than our goal, the SSC specification of $2440 \mathrm{~A} / \mathrm{mm}^{2}$ at $6.6 \mathrm{~T}$ and $4.2 \mathrm{~K}$.

When the D19B wire was examined with scanning electron microscopy, some upset of the billet and distortions of the filaments were noted, probably because of excess void space in the billet at the time of extrusion. Surprisingly, despite the distortion, no difficulties were encountered in drawing a single, unbroken piece of wire $3300 \mathrm{~m}$ long. After it was formed into cable and used to fabricate D19B, we found that its performance was quite close to expectations, with nearly the predicted magnetic field and little $J_{c}$ degradation. The distortion, upset, and sausaging problems all appear to be solvable through better process control, and Supercon, Inc., is proceeding with scale-up to full-sized production billets and optimization of $J_{c}$ in SSC-type wire, which we will test in D19C. Figure 4-7 shows a cross section of this wire.

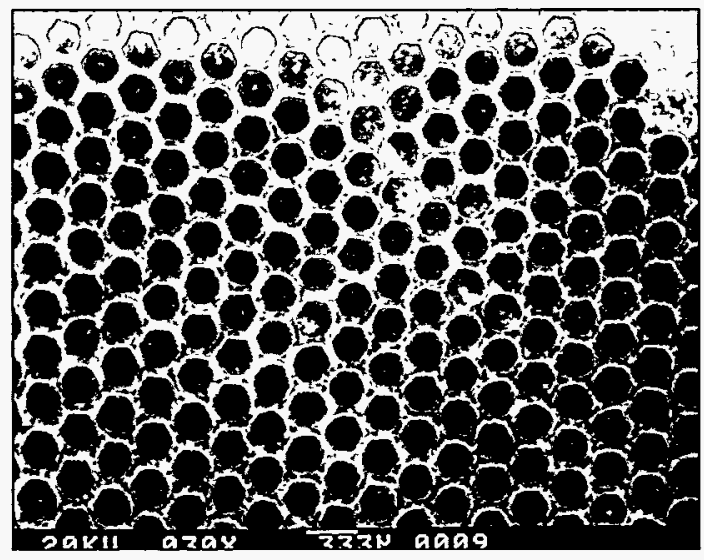

Figure 4-7. APC wire produced by Supercon, Inc., with a patented process in 1992 showed a uniform structure despite a local area ratio (copper matrix to superconductor filaments) of about 0.6 . It is desirable to make this ratio as low as possible to increase the critical current density. The whole wire is shown at top and an electron photomicrograph of an individual filament at bottom. Further scale-up to full-production-size billets is proceeding.

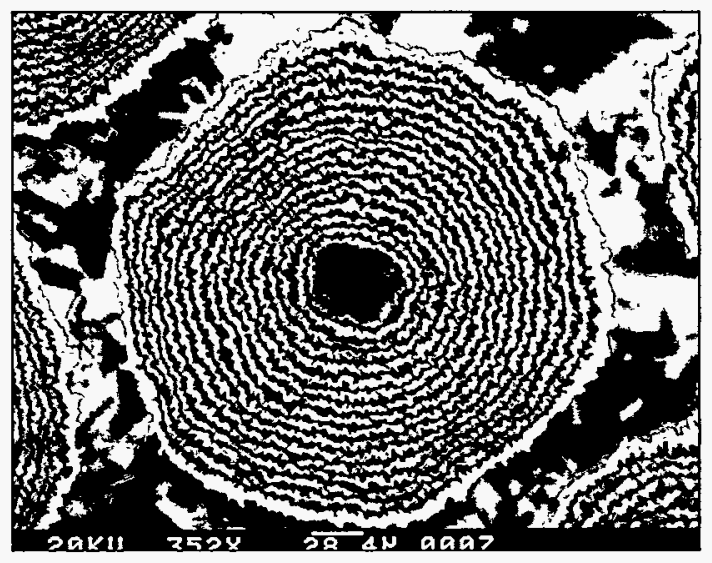

XBB 939-6074 
Until results are available from the scale-up to full-production-sized multifilamentary bullets, it will not be possible to make detailed projections of the cost savings expected from the use of APC materials. However, the raw materials are significantly cheaper, and the manufacturing process costs less because (among other factors) a variety of direct and indirect costs associated with heat treatment are obviated. Combining these savings, we estimate that the APC superconductor will be about half the cost of conventional $\mathrm{NbTi}$ of comparable specifications.

A longtime goal of our superconductor R\&D program has been to develop improved techniques and tooling for the fabrication of Rutherford-style cable. The R\&D cabling machine we developed to meet the needs of the SSC, and which is now available in a commercial version, continues to serve our R\&D needs. In 1993 we upgraded the machine (Figure 4-8) with a new spool and a powered Turk's-head so that it could fabricate cables with as

\author{
Cable and Cabling- \\ Machine Development
}
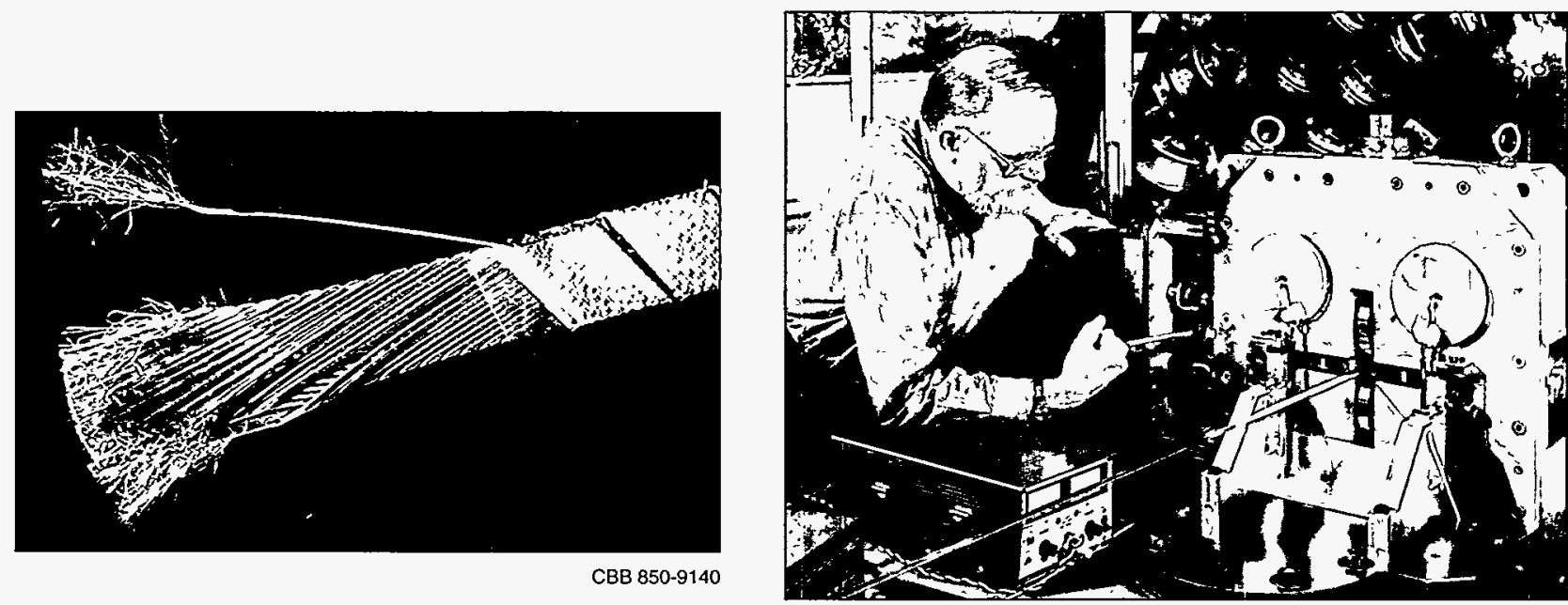

CBB 915-3356

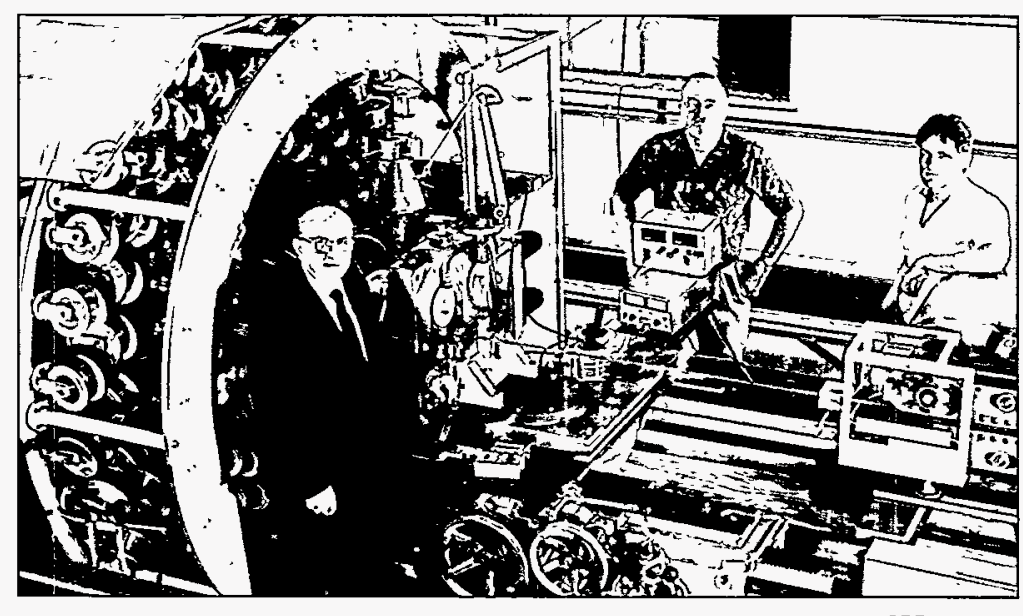

Figure 4-8. This cabling machine (whose designer was recognized in 1992 with the DOE Technology Transfer Excellence Award) adds greatly to our experimental program. It can produce cable with as many as 60 strands-about a third again as many as other cabling machines. Cable can be produced in a variety of cross sections, including keystoned and rectangular. A close-up of a representative cable shows how thousands of filaments a few tens of $\mu \mathrm{m}$ in diameter, made of superconductor in a copper matrix, are braided into strands of wire, which are then formed into a cable. 
High- $T_{c}$ Superconductor at Low Temperature many as 60 strands of superconducting wire. With this new equipment, we have been able to fabricate cables that are $30 \%$ wider and contain $25 \%$ more strands than the cable we developed in the late 1980s for the SSC dipole magnets. This 60-strand capability-nearly a third again as much as other machines-not only furthers cable manufacturing per se, but also, combined with the expert assistance of its operators, provides our magnet designers with additional flexibility in their choice of superconducting materials and cable designs. For example, we used it to make the rectangular 48-strand $\mathrm{Nb}_{3} \mathrm{Sn}$ cable for D20, and, while D20 is assembled, we are continuing to use the machine to determine how best to cable this material.

Although most of the emphasis in the program has been placed on the traditional Bardeen-Cooper-Shockley (BCS) superconductors, we also have a promising investigation into so-called high $-T_{c}$ superconductors. These materials, which are often used at liquid-helium temperatures in order to achieve high magnetic fields and maximum current density, might someday be useful for accelerator and other magnets. However, many challenges have to be overcome in order to turn them into useful engineering materials for devices such as accelerator magnets that must withstand great stress or must be built up into complicated shapes.

In an effort currently funded through the Laboratory-Directed Research and Development program (and interesting to at least one potential industrial partner), we are examining phase equilibria and microstructural texture in thick films of the bismuth oxide ( $\mathrm{BiSrCaCuO}$ ) family of compounds. In particular, we are studying ways of creating and preserving an aligned microstructure in the $\mathrm{Bi}_{2} \mathrm{Sr}_{2} \mathrm{Ca}_{1} \mathrm{Cu}_{2} \mathrm{O}_{2}$ or "2212" phase throughout the phase transformations that occur during processing, both within the bismuth oxide compound and between it and its silver substrate. Earlier work indicated $\mathrm{a} J_{\mathrm{c}}$ of greater than $1000 \mathrm{~A} / \mathrm{mm}^{2}$ for 2212 bismuth-oxide material. This figure seems to be on the same order as the $J_{\mathrm{c}}$ of NbTi conductor (albeit at a low magnetic field), although the sample was too short to be used in a magnet coil. Whether formed into tapes for windings or deposited onto surfaces as a coating (e.g., for superconducting rf cavities in accelerators), high- $T_{c}$ compounds represent a promising area of development.

Following up on earlier short-sample tests, we have completed a vertical furnace to process tapes of significant length. In 1994 we expect to fabricate a solenoid using tapes of 2212 on a silver substrate. 
A. Asner, "High field accelerator magnets beyond 10T; why, how and when," High Field Accelerator Magnet Workshop (Berkeley, California, 1993).

P. Barale, S. Caspi, W. Gilbert, M.I. Green, A. Lietzke, R. Schermer, and D. Van Dyke, "Magnetic measurements of the $5 \mathrm{~m} \mathrm{QC}$ series quadrupole at LBL," in Proceedings of the International Industrial Symposium on the Super Collider (San Francisco, CA, 1993); LBL-34052 (1993).

P. Barale, M.I. Green, W. Greidman, D. Hall, D. Lerner, J. MacFarland, R. Schermer, and D. Van Dyke, "A modern harmonic error analysis measurement system for dipole and quadrupole magnets at LBL, " in Proceedings of the Magnet Technology Conference (Victoria, B.C., Canada, 1993); LBL-33476 (1993).

S. Caspi, "The use of harmonics in 3D magnetic fields," in Proceedings of the Magnet Technology Conference (Victoria, B.C., Canada, 1993); LBL-33432 (1993).

D. Dell'Orco, "High field dipole magnet utilizing a block design," High Field Accelerator Magnet Workshop (Berkeley, CA, 1993).

D. Dell'Orco, "High field dipole magnet utilizing a cosine theta design," High Field Accelerator Magnet Workshop (Berkeley, CA, 1993).

D. Dietderich and M. Walker, "Progress in the development of BSCCO-2212

superconducting," in Proceedings of the 5th U.S./Japan Workshop on High Tc

Superconductor (Tsukuba, Japan, 1992); LBL34662 (1992).

A. Lietzke, R. Armer, P. Bish, J. Rechen, R. Schafer, and C. Taylor, "Upgrading the LBL magnet test facility's $1.8 \mathrm{~K}$ subatmospheric refrigerator system," in Proceedings of the Cryogenic Engineering Conference (Albuquerque, NM, 1993); LBL-33455 (1993).
A. Lietzke, P. Barale, B. Benjegerdes, S. Caspi, J. Cortella, D. Dell'Orco, W. Gilbert, M. Green, K. Mirk, C. Peters, R. Scanlan, C. Taylor, and A. Wandesforde, "SSC quadrupole magnet performance at LBL," in Proceedings of the International Industrial Symposium on the Super Collider (San Francisco, CA, 1993); LBL-33002 (1993).

J. Royet and R. Scanlan, "Recent developments in superconducting cabling technology," LBL-33440 (1993).

R. Scanlan, "Fabrication and testing of $\mathrm{Nb}_{3} \mathrm{Sn}$ cables for dipole D20," High Field Accelerator Magnet Workshop (Berkeley, CA, 1993).

R. Scanlan, "Progress in fabrication of artificial pinning center conductors," High Field Accelerator Magnet Workshop (Berkeley, CA, 1993).

R. Scanlan, "Technology for higher field, higher current density superconductors," invited paper in Proceedings of the Particle Accelerator Conference (Washington, D.C., 1993); LBL-33373 (1993).

R. Scanlan, C. Taylor, J. Royet, A. Lietzke, and A. Wandesforde, "Evaluation of APC $\mathrm{NbTi}$ superconductor in a model dipole magnet," in Proceedings of the Magnet Technology Conference (Victoria, B.C., Canada, 1993); LBL-33439 (1993).

J.M. van Oort, R.M. Scanlan, H.W. Weijers, and H.H.J. ten Kate, "The reduction of the critical current under transverse pressure in a new TWCA MJR cabled conductor," in Proceedings of the Cryogenic Engineering Conference (Albuquerque, NM, 1993); LBL33430 (1993).

J. van Oort and H. ten Kate, "A fiber optics sensor for strain \& stress measurement in superconducting accelerator magnets," in Proceedings of the Magnet Technology Conference (Victoria, BC, Canada, 1993); LBL-33431 (1993).
Publications and Presentations 